Ann. Biol. anim. Bioch. Biophys., I970, 10 (2), 239-252.

\title{
FACTEURS NUTRITIONNELS MODIFIANT LE POIDS DE L'GEUF ET DE SES CONSTITUANTS
}

\author{
B. LECLERCQ \\ avec la collaboration technique de Marie-Rose Salichov \\ Station de Recherches avicoles, \\ Centre de Recherche de Tours, 37 - Nouzilly \\ Institut national de la Recherche agronomique
}

\section{RÉSUMÉ}

Les effets de plusieurs facteurs nutritionnels pouvant intervenir pour diminuer le poids de l'cuuf, ont été analysés.

La présence d'acides gras de forme trans dans le régime de la Poule pondeuse provoque une réduction rapide du poids de l'œuf qui porte surtout sur le vitellus, bien que la durée de la phase de grand accroissement soit augmentée. Ces effets sont indépendants de la teneur du régime en acide linoléique. Ils s'accompagnent d'une modification de la composition du jaune.

La distribution d'un régime déficient en acides aminés indispensables (méthionine et lysine) conduit à une réduction notable du poids de l'œuf qui, cette fois, intéresse surtout l'albumen dont la composition est modifiée plus profondément que celle du jaune.

L'incorporation d'huile de colza contenant de l'acide érucique au régime de la Poule conduit en quelques jours à une réduction đu poids de l'œuf portant à la fois sur le jaune et sur le blanc. Cet effet se manifeste à de faibles taux d'incorporation. Il disparaît en quelques jours lorsque cesse l'ingestion d'huile de colza. Il n'apparaît pas si l'on utilise une huile de colza dépourvue d'acide érucique.

L'existence de sites de synthèse distincts pour les constituants du jaune et du blanc de l'œuf explique leur plus ou moins grande sensibilité aux différents facteurs nutritionnels.

\section{INTRODUCTION}

De nombreux travaux récents ont mis en évidence l'effet favorable de l'acide linoléique sur le poids de l'œuf (Shutze et Jensen, I963; Calvert I965). Pour notre part, nous avons montré que d'autres acides gras du régime de la Poule peuvent modifier profondément le poids de l'œuf : les acides gras trans réduisent la vitellogenèse et le poids de l'œuf (LECLERCQ et BLUM, I967). Nous tentons d'approfondir l'étude des phénomènes précédemment observés en comparant les régimes contenant 
de l'huile élaïdisée à des régimes renfermant d'autres matières grasses ou déficients en acides aminés indispensables.

\section{MATÉRIEL ETT MÉTHODES}

\section{Animaux et régimes}

Nous rapportons ici les résultats de 4 essais différents. Tous les animaux, âgés de 8 à 2 mois, sont issus d'un croisement commercial de Rhode Island Red $\times$ Wyandotte. Ils sont nourris et abreuvés à volonté et soumis à une période journalière d'éclairement de 16 heures.

Chaque essai comporte 2 ou 3 périodes :

- période préexpérimentale ( 28 jours) pendant laquelle tous les animaux reçoivent le même régime témoin ; elle constitue une base de référence pour chaque sujet.

— période expérimentale ( 28 jours) qui débute par la constitution de lots aussi homogènes que possible du point de vue de la ponte. Le lot témoin reçoit toujours le même régime distribué pendant la période préexpérimentale. Dans tous les lots expérimentaux, on modifie le régime pour apprécier l'effet des facteurs nutritionnels étudiés.

- période post-expérimentale (28 jours) qui n'existe que dans les essais 2 et 4 . Au cours de cette période, tous les animaux reçoivent à nouveau le régime témoin. On peut ainsi comparer les résultats des lots expérimentaux à ceux du lot témoin et constater si les effets des facteurs nutritionnels se poursuivent au-delà de la période expérimentale.

Pour chaque période, on considère que les I 4 premiers jours correspondent à une phase d'adaptation et on effectue au cours des I 4 derniers jours les différentes mesures : nombre d'œufs, poids de l'œuf entier, celui du jaune et de la coquille, poids vif des animaux, consommation d'aliment. En outre, c'est pendant ces 14 derniers jours que sont réalisées quelques analyses ou mesures particulières à certains essais qui seront précisées par la suite.

Les différents essais sont tous réalisés selon le même schéma expérimental. Ils ne diffèrent que par la nature des régimes distribués.

\section{Essai $n^{0} 1$.}

Il comporte 6 lots de 4 poules. La composition des régimes figure dans le tableau I.

Les lots sont désignés par le sigle du régime qu'ils reçoivent pendant la période expérimentale :

Lot $\mathrm{T}$ : régime témoin $\mathrm{T}$.

Lot I : régime I contenant I 5 p. too d'huile d'arachide isomérisée.

Lot IL : régime IL contenant I5 p. Ioo d'huile d'arachide isomérisée et un supplément d'acide linoléique sous forme d'huile de maïs.

Lot $\mathrm{S}$ : régime $\mathrm{S}$, contenant $\mathrm{I}_{5} \mathrm{p}$. Ioo de suif " premier jus ».

Lot A : régime A contenant I5 p. Ioo d'huile d'arachide pure.

Lot VB : régime VB identique au lot témoin mais non supplémenté en méthionine et lysine.

Tous les régimes sont isoénergétiques et renferment une partie commune destinée à l'apport des protéines non équilibrées, des minéraux et des vitamines. Les régimes T, I, IL, S et A supplémentés en méthionine et en lysine synthétiques contiennent respectivement $0,32 \mathrm{p}$. Ioo et 0,70 p. I oo de chacun de ces acides aminés. Le régime VB non supplémenté ne titre que o, I 9 p. Ioo de méthionine et 0,55 p. Ioo de lysine. Pour tous les autres acides aminés indispensables, les teneurs de tous les régimes correspondent aux normes classiquement admises. La composition en acides gras des matières grasses figure dans le tableau 2.

\section{Essai no 2 .}

On ne conserve du protocole précédent que les lots T, I et VB. A cet effet sont constitués 3 lots de 5 poules. Il n'y a pas de post-expérience. En outre, on prélève en vue d'analyses un mélange de jaune et un mélange de blanc provenant de trois oufs de chaque poule et pour chaque période. Enfin, sur les animaux du lot $\mathrm{I}$ et ceux du lot $\mathrm{T}$ on mesure la durée de la phase de grand accroissement de l'ovocyte au cours de la préexpérience et de l'expérience : on a recours pour cela à la méthode des colorants en employant le Noir Crésol (1) et le Rouge Écarlate $R\left({ }^{1}\right)$ (LACASSAGNE, 1957).

(1) Produits R. A, L. Kuhlman, Paris. 
TABLEAU I

Essais $n^{\circ} 1$ et $n^{\circ} 2$

Composition des régimes utilisés

(en p. roo)

\begin{tabular}{|c|c|c|c|c|c|c|}
\hline & Lot $\mathrm{T}$ & Lot $I$ & Lot $1 \mathrm{~L}$ & Lot A & Lot $\mathrm{S}$ & Lot VB \\
\hline $\begin{array}{l}\text { Concentré protéique minéral et } \\
\text { vitaminique }(\mathbf{1}) \ldots \ldots \ldots \ldots \ldots\end{array}$ & 52,2 & 52,2 & 52,2 & 52,2 & 52,2 & 52,2 \\
\hline Amidon $\ldots \ldots \ldots \ldots \ldots \ldots$ & 37,5 & 10,0 & 0 & 10,0 & 15,9 & 37,5 \\
\hline Cellulose . . . . . . . . . . & 6,0 & 22,5 & 28,5 & 22,5 & 16,6 & 6,0 \\
\hline Huile d'arachide isomérisée ... & 0 & 15,0 & 15,0 & 0 & 0 & 0 \\
\hline Huile de maïs $\ldots \ldots \ldots \ldots$ & 4,0 & 0 & 4,0 & 0 & 0 & 4,0 \\
\hline Huile d'arachide naturelle .... & 0 & 0 & 0 & 15,0 & 0 & 0 \\
\hline Suif $\ldots \ldots \ldots \ldots \ldots \ldots \ldots$ & 0 & 0 & 0 & 0 & 15,0 & 0 \\
\hline DL-méthionine . . . . & 0,13 & 0,13 & 0,13 & 0,13 & 0,13 & 0 \\
\hline L-Lysine $\ldots \ldots \ldots \ldots \ldots \ldots$ & 0,15 & 0,15 & 0,15 & 0,15 & 0,15 & 0 \\
\hline $\begin{array}{c}\text { É nergie métabolisable }{ }^{(2)} \\
(\mathrm{kcal} / \mathrm{kg}) \quad \ldots \ldots \ldots \ldots \ldots \ldots\end{array}$ & 2900 & 2900 & 2900 & 2900 & 2900 & 2900 \\
\hline Taux protidique $(\mathrm{p} .100) \ldots \ldots$ & 16,8 & 16,8 & 16,8 & 16,8 & 16,8 & 16,6 \\
\hline Teneur en lysine (p. 100)..... & 0,704 & $0,70 /$ & 0,701 & $0,70_{\mathrm{t}}^{\prime}$ & 0,704 & 0,554 \\
\hline Teneur en méthionine (p. 100). & 0,318 & 0,318 & 0,318 & 0,318 & 0,318 & 0,188 \\
\hline
\end{tabular}

( $\left.{ }^{1}\right)$ Composition du concentré protéique, minéral et vitaminique : tourteau d'arachide : 59 p. 100 , blé : 19 p. 100 , amidon de maïs : 2,9 p. 100, carbonate de calcium : 13,4 p. 100, phosphate bicalcique : 3,8 p. 100 , bicarbonate de sodium : 0,8 p. 100 , sulfate de cuivre : 20 p.p.m., carbonate de magnésium : 240 p.p.m., sulfate de fer : 220 p.p.m., sulfate de zinc : 450 p.p.m., sulfate de manganèse : 600 p.p.m., oxyde de magnésie : 300 p.p.m.

Vitamine A : $30000 \mathrm{UI} / \mathrm{kg}$. Vitamine $\mathrm{D}_{3}: 4000 \mathrm{UI} / \mathrm{kg}$. Riboflavine : $7,7 \mathrm{mg} / \mathrm{kg}$. Choline : $2,90 \mathrm{~g} / \mathrm{kg}$. BHT : $0,19 \mathrm{~g} / \mathrm{kg}$.

(2) Calculées d'après les tables d'analyses.

TABLEAU 2

Composition en acides gras des lipides alimentaires

(en p. roo des acides gras totaux)

\begin{tabular}{|c|c|c|c|c|c|}
\hline Acide gras & $\begin{array}{l}\text { Huile } \\
\text { d'arachide } \\
\text { isomérisée }\end{array}$ & $\begin{array}{c}\text { Huile } \\
\text { d'arachide } \\
\text { naturelle }\end{array}$ & Suif & $\begin{array}{c}\text { Huile } \\
\text { de colza } \\
\text { normale }\end{array}$ & $\begin{array}{l}\text { Huile de colza } \\
\text { sans acide } \\
\text { érucique }\end{array}$ \\
\hline $\begin{aligned} 16 & : 0 \\
18 & : 0 \\
18 & : 1 \text { cis } \\
18 & : 1 \text { trans } \\
18 & : 2 \text { (1) } \\
18: & 3 \\
20 & : 1 \\
22 & : 1 \\
\text { Autres } & \text { acides gras }\end{aligned}$ & $\begin{array}{r}13,3 \\
3,8 \\
14,5 \\
49,5 \\
11,2\end{array}$ & $\begin{array}{r}13,0 \\
2,0 \\
70,0 \\
\\
14,0 \\
1,0\end{array}$ & $\begin{array}{r}29,0 \\
25,8 \\
34,2 \\
\\
2,0\end{array}$ & $\begin{array}{r}3,5 \\
1,2 \\
13,9 \\
\\
14,0 \\
8,1 \\
9,7 \\
49,6\end{array}$ & $\begin{array}{r}5,0 \\
2,0 \\
60,0 \\
21,0 \\
10,0 \\
1,0 \\
1,0\end{array}$ \\
\hline
\end{tabular}

(1) Ensemble des isomères cis-cis, cis-trans, trans-cis et trans-trans pour l'huile isomérisée. 
Essai $n^{\circ} 3$.

Chaque lot renferme 5 poules. Le lot témoin est le lot $\mathrm{C}_{0}$. Les lots ingèrent pendant la période expérimentale les régimes $\mathrm{C}_{0}, \mathrm{C}_{2}, \mathrm{C}_{5}$ et $\mathrm{C}_{8}$ renfermant $0,2,5$ et $8 \mathrm{p}$. Ioo d'huile de colza riche en acide érucique. La composition des régimes figure dans le tableau 3 .

TABLEAU 3

Essais $n^{\circ} 3$ et 4

Composition des régimes utilisés

\begin{tabular}{|c|c|c|c|c|c|}
\hline & $\mathrm{C}_{0}$ & $\mathrm{C}_{2}$ & $\mathrm{C}_{5}$ & $\mathrm{C}_{8}$ & $\mathrm{C}_{8}$ bis \\
\hline Maïs broyé $\ldots \ldots \ldots \ldots \ldots \ldots \ldots \ldots$ & 50,0 & 50,0 & 50,0 & 50,0 & 50,0 \\
\hline Tourteau de soja $\ldots \ldots \ldots \ldots \ldots \ldots \ldots$ & 14,0 & 14,0 & 14,0 & 14,0 & 14,0 \\
\hline Farine de luzerne déshydratée $\ldots \ldots \ldots \ldots$ & 5,0 & 5,0 & 5,0 & 5,0 & 5,0 \\
\hline Farine de poisson $\ldots \ldots \ldots \ldots \ldots \ldots$ & 6,0 & 6,0 & 6,0 & 6,0 & 6,0 \\
\hline Carbonate de calcium $\ldots \ldots \ldots \ldots \ldots$ & 7,0 & 7,0 & 7,0 & 7,0 & 7,0 \\
\hline Phosphate bicalcique................ & 2,0 & 2,0 & 2,0 & 2,0 & 2,0 \\
\hline Mélange oligo-minéral ${ }^{(1)} \ldots \ldots \ldots \ldots \ldots$ & 0,25 & 0,25 & 0,25 & 0,25 & 0,25 \\
\hline Mélange vitaminique ${ }^{(2)} \ldots \ldots \ldots \ldots \ldots \ldots$ & 0,75 & 0,75 & 0,75 & 0,75 & 0,75 \\
\hline Amidon $\ldots \ldots \ldots \ldots \ldots \ldots \ldots \ldots$ & 15,0 & 11,3 & 5,6 & 0 & 0 \\
\hline Cellulose $\ldots \ldots \ldots \ldots \ldots \ldots \ldots \ldots \ldots \ldots$ & 0 & 1,7 & 4,4 & 7,0 & 7,0 \\
\hline Huile de colza contenant l'acide érucique ... & 0 & 2,0 & 5,0 & 8,0 & 0 \\
\hline Huile de colza sans acide érucique $\ldots . . .$. & 0 & 0 & 0 & 0 & 8,0 \\
\hline
\end{tabular}

(1) Composition du complément minéral (pour $250 \mathrm{~g}$ ) : Bicarbonate de sodium : $100 \mathrm{~g}$, sulfate de cuivre : $1 \mathrm{~g}$, iodure de potassium : $0,2 \mathrm{~g}$, sulfate de zinc : $22 \mathrm{~g}$, sulfate de manganèse : $30 \mathrm{~g}$, oxyde de magnésium : $15 \mathrm{~g}$, carbonate magnésium : $12 \mathrm{~g}$, carbonate de calcium : $70 \mathrm{~g}$.

${ }^{(2)}$ Composition du complément vitaminique (pour $750 \mathrm{~g}$ ): vitamine $\mathrm{A}: 1600000 \mathrm{UI}$, vitamine $\mathrm{D}_{\mathbf{3}}$ : $200000 \mathrm{UI}$, riboflavine $: 400 \mathrm{mg}$, pantothénate de calcium : $1 \mathrm{~g}$, nicotinamide : $2 \mathrm{~g}$, tocophénol : 1200 UI BHT : $10 \mathrm{~g}$, DL-méthionine : $100 \mathrm{~g}$, choline : $150 \mathrm{~g}$, avoine broyée : $97 \mathrm{p} .750 \mathrm{~g}$.

L'introduction d'huile et de cellulose est réalisée aux dépens de l'amidon de façon à maintenir égaux les taux énergétiques de tous les régimes.

\section{Essai $n^{\circ} 4$.}

Cet essai permet de comparer les effets de l'huile de colza selon qu'elle contient ou non de l'acide érucique. Le lot témoin (lot $\mathrm{C}_{0}$ ) reçoit le régime $\mathrm{C}_{0}$ durant tout l'essai. Les lots expérimentaux sont les lots $\mathrm{C}_{8}$ ingérant un aliment contenant $8 \mathrm{p}$. Ioo d'huile de colza riche en acide érucique et le lot $\mathrm{C}_{8}$ bis qui reçoit le régime $\mathrm{C}_{8}$ bis renfermant $8 \mathrm{p}$. Ioo d'huile de colza très pauvre en acide érucique. La composition des régimes fait l'objet du tableau 3.

\section{Méthodes analytiques}

- Pesée des constituants de l'œuf.

Le poids du jaune est déterminé directement; celui du blanc est obtenu en soustrayant du poids d'œuf entier celui du jaune et celui de la coquille lavée et séchée.

- Dosages des constituants de l'œuf (essai 2).

La teneur en protéines est déterminée par la méthode de Kjeldahl. Celle des lipides a près extraction, selon la méthode de Folch.

- Analyse statistique.

Elle porte sur les différences entre 6 données de la période considérée et la moyenne de 6 données de la période de référence (préexpérience). 


\section{TABLEAU 4}

Essai $n^{\circ} 1$

(Huile isomérisée et valeur biologique des protéines) Influence des différents régimes sur le poids de l'auf du vitellus et de l'albumen

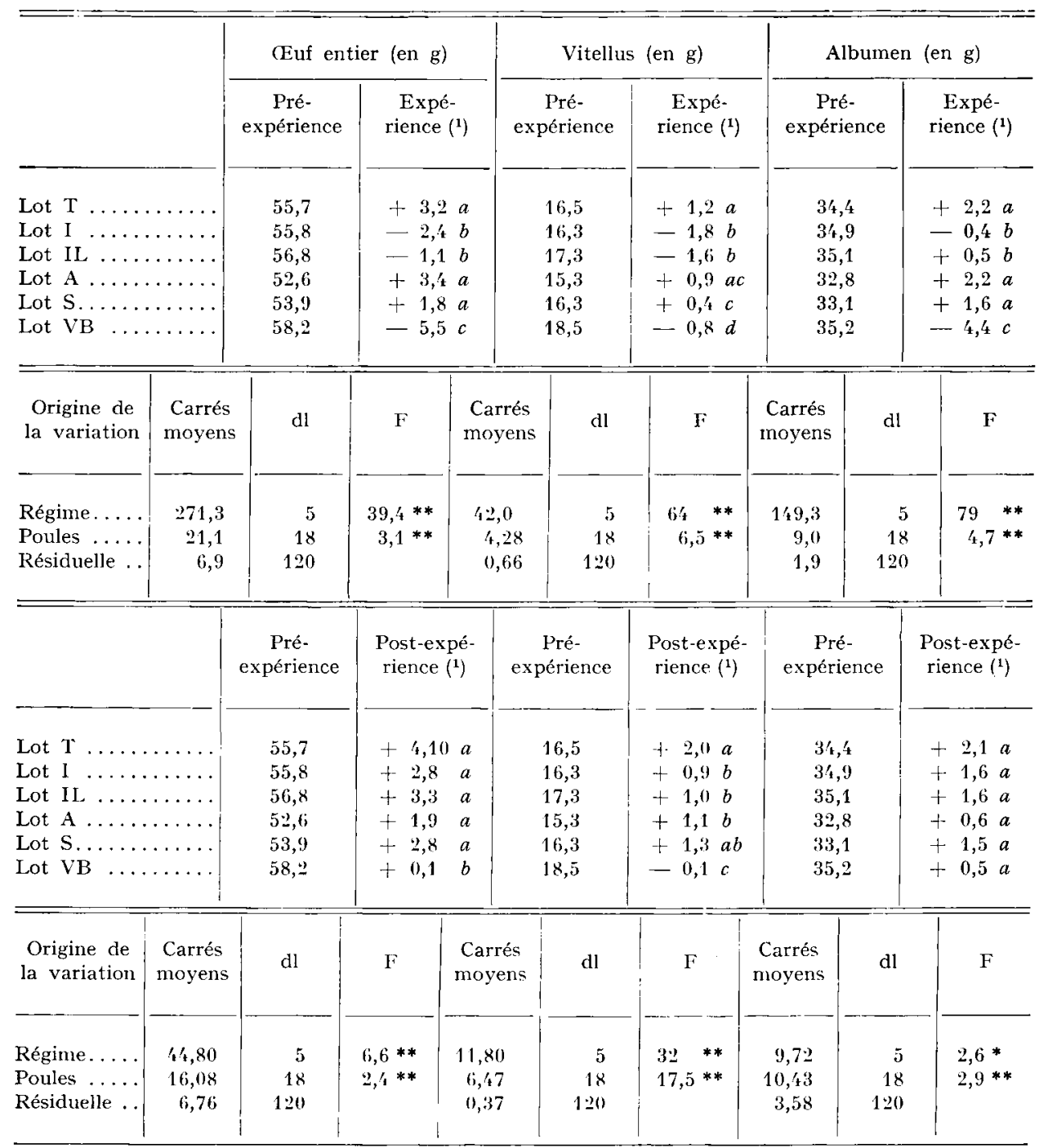

(1) Les valeurs figurant dans cette colonne sont les variations par rapport à la préexpérience ; accompagnées d'une même lettre, elles ne sont pas significativement différentes au seuil 0,01 .

** Effet significatif au seuil 0,01 . * Effet significatif au seuil 0,05 . 


\section{RÉSULTATS}

\section{Influence de l'huile isomérisée sur le poids et la composition de l'œuf et la durée de développement du follicule}

Le tableau 4 indique les variations de poids de l'œuf entier, du vitellus et de l'albumen entre la préexpérience, l'expérience et la post-expérience de l'essai $n^{\circ} \mathrm{I}$. Chez tous les animaux ingérant de l'huile isomérisée, on constate une réduction importante du poids de l'œuf quel que soit l'apport alimentaire d'acide linoléique (lots I et IL). La réduction intéresse presqu'exclusivement le vitellus. Le poids de l'albumen, lui, varie peu; toutefois celui-ci ayant augmenté notablement dans le lot témoin pendant le même temps, l'évolution du poids du blanc est significativement moins importante pour les lots recevant de l'huile isomérisée que pour le lot témoin.

Le lot recevant I5 p. Ioo d'huile d'arachide non isomérisée présente une évolution générale très voisine de celle du lot $T$ (témoin). Pour le lot $\mathrm{S}$ ( 5 p. Ioo de suif) les variations de poids de l'œuf et de l'albumen ne diffèrent pas de celles du lot $T$; seul le poids du jaune augmente un peu moins vite. Dans tous les cas, l'effet "Poule " est significatif et révèle l'importance des réactions individuelles.

La confrontation des résultats de la post-expérience avec ceux de la préexpérience permet de constater que, lorsqu'ils sont nourris à nouveau avec l'aliment témoin, les animaux des lots expérimentaux tendent à compenser leur retard sur le lot témoin : il en est ainsi du poids moyen de l'œuf et de l'albumen dont les variations ne sont pas significativement différentes. La compensation n'est pas tout à fait complète pour le vitellus chez les lots I, II, et A.

Dans le tableau 5 sont rapportés les résultats de $1^{\prime}$ essai $n^{\circ} 2$.
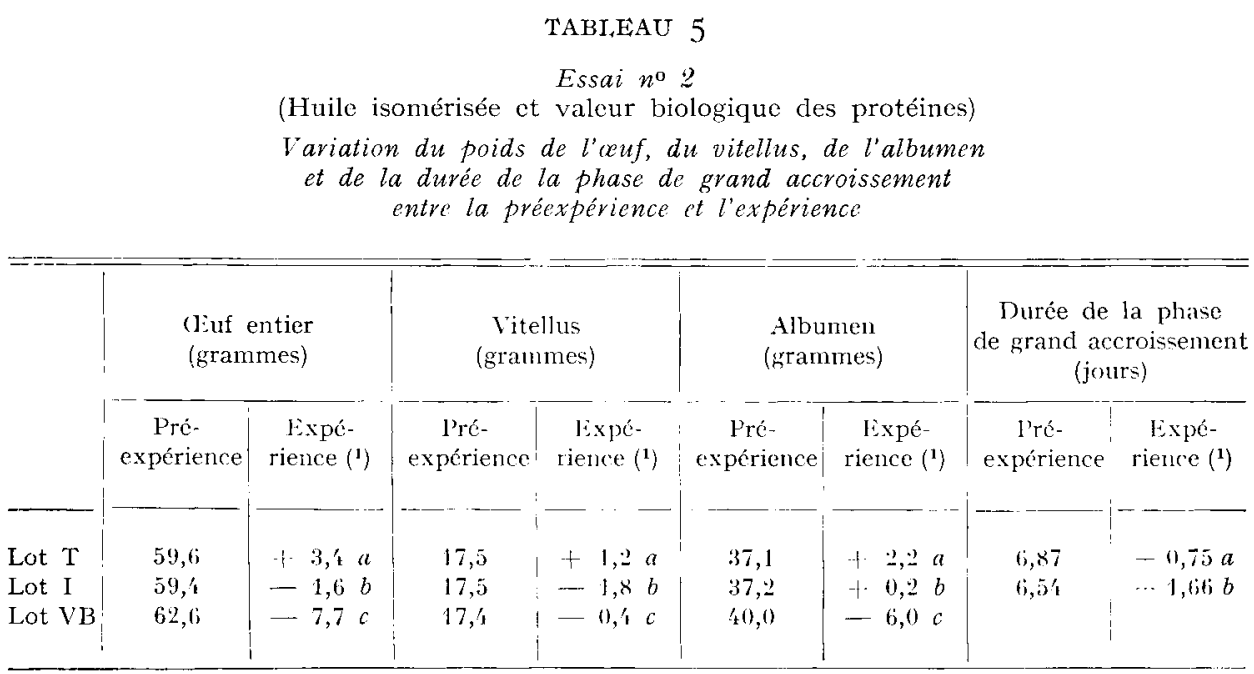

(1) Les valeurs figurant dans cette colonne sont les variations par rapport à la pré-expérience, accompagnées d'une même lettre, elles ne sont pas significativement différentes au seuil 0,01 . 
Les mêmes constatations que dans l'essai $n^{0}$ I peuvent être faites pour le poids de l'œuf, du vitellus et de l'albumen. On observe de plus que la durée de la phase de grand accroissement de l'ovocyte s'allonge de 1,66 jour au cours de l'expérience chez les animaux du lot I et de 0,75 jour pour le lot témoin. Cette différence voisine d'une journée est significative.

On peut enfin constater, grâce au tableau 6 que l'ingestion d'huile d'arachide isomérisée modifie la composition du vitellus de l'œuf : davantage de protéines, moins de lipides. Aucune modification n'est observée dans le blanc.

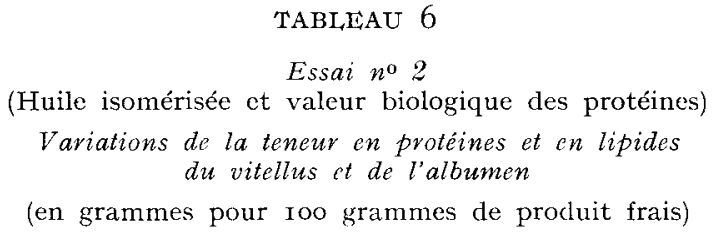

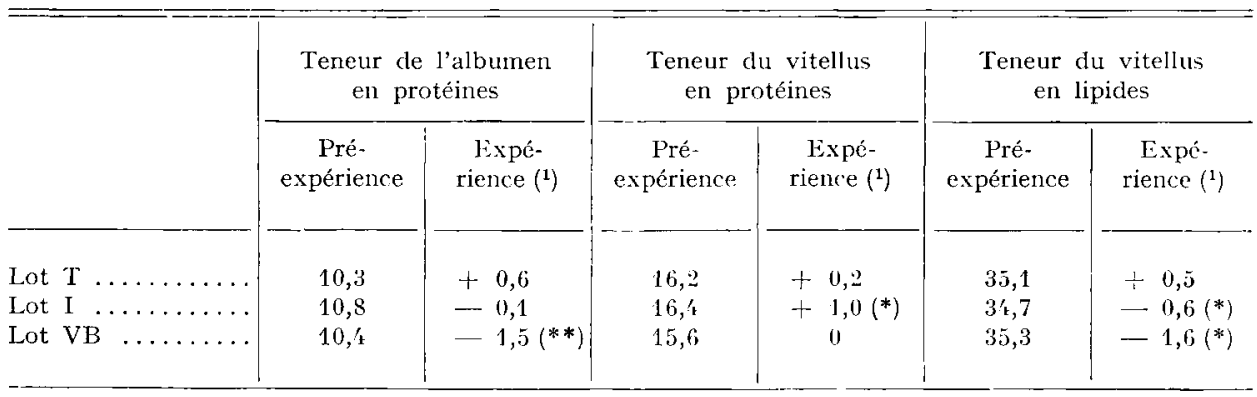

(i) Les valeurs figurant dans cette colonne sont les variations par rapport à la pré-expérience.

(*) Variation entre pré-expérience et expérience significative au seuil 0,05 .

(**) Variation entre pré-expérience et expérience significative au seuil 0,01 .

\section{Infuence de la déficience en méthionine et lysine sur le poids et la composition de l'auf}

Les lots VB (essais nos I et 2 ) permettent d'apprécier l'effet d'une déficience en méthionine et 1ysine (tabl. 4 et 5). On constate une réduction du poids de 1'œuf qui porte surtout sur le blanc $\left(-4,4 \mathrm{~g}\right.$ dans l'essai $\mathrm{n}^{0} \mathrm{I},-6, \mathrm{o}$ g dans l'essai $\left.\mathrm{n}^{0} 2\right)$; la taille du jaune est peu modifiée $(-0,8 \mathrm{~g}$ et $-0,4 \mathrm{~g})$.

La composition du jaune et du blanc des cufs du lot VB est présentée dans le tableau 6. Les deux constituants de l'œuf sont altérés. Dans le blanc, la teneur en protéines accuse une chute spectaculaire; quant au jaune, si sa teneur en protéines ne varie pas, sa concentration en lipides décroît.

\section{Influence de l'huile de colza sur le poids de l'auf et de ses constituants}

Dans le tableau 7 , nous indiquons les variations que subissent le poids de l'œuf, celui du vitellus et celui de l'albumen sous l'effet de doses différentes d'huile de colza 


\section{TABLEAU 7}

Essai $n^{0} 3$

(Différentes doses d'huile de colza)

Variations du poids de l'cuf, l'albumen et du vitellus entre la préexpérience, l'expérience et la post-expérience

\begin{tabular}{|c|c|c|c|c|c|c|c|c|c|c|}
\hline \multirow{2}{*}{\multicolumn{2}{|c|}{ Lot }} & \multicolumn{3}{|c|}{ Cuf entier (en g) } & \multicolumn{3}{|c|}{ Vitellus (en g) } & \multicolumn{3}{|c|}{ Albumen (en g) } \\
\hline & & $\begin{array}{c}\text { Pré- } \\
\text { expérience }\end{array}$ & \multicolumn{2}{|c|}{$\begin{array}{l}\text { Expé- } \\
\text { rience (1) }\end{array}$} & \multicolumn{2}{|c|}{$\begin{array}{c}\text { Pré- } \\
\text { expérience }\end{array}$} & $\begin{array}{l}\text { Expé- } \\
\text { rience }{ }^{(1)}\end{array}$ & \multicolumn{2}{|c|}{$\begin{array}{c}\text { Pré- } \\
\text { expérience }\end{array}$} & $\begin{array}{l}\text { Expé- } \\
\left.\text { rience ( }{ }^{1}\right)\end{array}$ \\
\hline \multicolumn{2}{|c|}{ 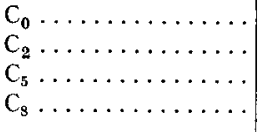 } & $\begin{array}{l}55,49 \\
55,88 \\
55,57 \\
55,83\end{array}$ & \multicolumn{2}{|c|}{$\begin{array}{l}+1,37 a \\
+\quad 0,86 a \\
-2,27 b \\
-5,30 c\end{array}$} & \multicolumn{2}{|c|}{$\begin{array}{l}15,57 \\
15,31 \\
15,08 \\
15,45\end{array}$} & $\begin{array}{l}+1,09 a \\
+\quad 0,56 a \\
-0,91 b \\
-1,49 c\end{array}$ & \multicolumn{2}{|c|}{$\begin{array}{l}35,10 \\
35,41 \\
35,50 \\
35,46\end{array}$} & $\begin{array}{l}+0,36 a \\
+\quad 0,31 a \\
-1,10 b \\
-2,83 c\end{array}$ \\
\hline $\begin{array}{c}\text { Origine de } \\
\text { la variation }\end{array}$ & $\begin{array}{l}\text { Carrés } \\
\text { moyens }\end{array}$ & $\mathrm{dl}$ & F & \multicolumn{2}{|c|}{$\begin{array}{l}\text { Carrés } \\
\text { moyens }\end{array}$} & $\mathrm{dl}$ & $\mathrm{F}$ & $\begin{array}{l}\text { Carrés } \\
\text { moyens }\end{array}$ & $\mathrm{dl}$ & $\mathrm{F}$ \\
\hline $\begin{array}{l}\text { Régime..... } \\
\text { Poule ...... } \\
\text { Résiduelle .. }\end{array}$ & $\begin{array}{c}33,3 \\
13,2 \\
1,94\end{array}$ & $\begin{array}{r}3 \\
16 \\
99\end{array}$ & $\begin{array}{r}17,2 * * \\
6,8 * * *\end{array}$ & \multicolumn{2}{|c|}{$\begin{array}{l}45,0 \\
2,90 \\
0,57\end{array}$} & $\begin{array}{r}3 \\
16 \\
99\end{array}$ & $\begin{array}{l}79 * * \\
5,1 * *\end{array}$ & $\begin{array}{l}83,7 \\
6,60 \\
1,01\end{array}$ & $\begin{array}{r}3 \\
16 \\
99\end{array}$ & $\begin{array}{l}83 * * \\
6,5 * *\end{array}$ \\
\hline & & $\begin{array}{c}\text { Pré- } \\
\text { expérience }\end{array}$ & \multicolumn{2}{|c|}{$\begin{array}{l}\text { Post-expé- } \\
\text { rience (1) }\end{array}$} & \multicolumn{2}{|c|}{$\begin{array}{c}\text { Pré- } \\
\text { expérience }\end{array}$} & $\begin{array}{l}\text { Post-expé- } \\
\text { rience }\left({ }^{1}\right)\end{array}$ & \multicolumn{2}{|c|}{$\begin{array}{c}\text { Pré- } \\
\text { expérience }\end{array}$} & $\begin{array}{l}\text { Post-expé- } \\
\text { rience }\left(^{(1}\right)\end{array}$ \\
\hline $\begin{array}{l}c_{0} \ldots \ldots \ldots \\
c_{2} \ldots \ldots \ldots \\
c_{5} \ldots \ldots \ldots \\
c_{8} \ldots \ldots \ldots\end{array}$ & $\begin{array}{l}\ldots \\
\cdots \\
\cdots \\
\cdots \cdots\end{array}$ & $\begin{array}{l}55,49 \\
55,88 \\
55,57 \\
55,83\end{array}$ & $\begin{array}{l}+\quad 2,0 \\
+\quad 3,7 \\
+\quad 2,5 \\
+\quad 3,0\end{array}$ & $\begin{array}{l}a \\
b \\
a \\
a\end{array}$ & & & $\begin{array}{l}+2,18 a \\
+1,86 a \\
+1,85 a \\
+\quad 2,01 a\end{array}$ & $\begin{array}{l}35, \\
35, \\
35, \\
35,\end{array}$ & & $\begin{array}{l}-0,20 a \\
+1,61 b \\
+0,48 a c \\
+1,15 b c\end{array}$ \\
\hline $\begin{array}{l}\text { Origine de } \\
\text { la variation }\end{array}$ & $\begin{array}{l}\text { Carrés } \\
\text { moyens }\end{array}$ & dl & $\mathrm{F}$ & $\begin{array}{l}\text { Car } \\
\text { moy }\end{array}$ & & $\mathrm{d} 1$ & $\mathrm{~F}$ & $\begin{array}{l}\text { Carrés } \\
\text { moyens }\end{array}$ & $\mathrm{dl}$ & $\mathrm{F}$ \\
\hline $\begin{array}{l}\text { Régime..... } \\
\text { Poule ...... } \\
\text { Résiduelle . . }\end{array}$ & $\begin{array}{r}15,0 \\
10,1 \\
2,7\end{array}$ & $\begin{array}{r}3 \\
16 \\
99\end{array}$ & $\begin{array}{l}5,6 * * \\
3,7 * *\end{array}$ & $\begin{array}{l}0, \\
1, \\
0,\end{array}$ & & $\begin{array}{r}3 \\
16 \\
99\end{array}$ & $\begin{array}{l}0,99 \\
1,80 *\end{array}$ & $\begin{array}{r}18,7 \\
4,30 \\
2,27\end{array}$ & $\begin{array}{r}3 \\
16 \\
99\end{array}$ & $\begin{array}{l}8,21 * * \\
1,90 *\end{array}$ \\
\hline
\end{tabular}

(1) Les valeurs figurant dans cette colonne sont les variations par rapport à la préexpérience ; accompagnées de la même lettre, elles ne sont pas significativement différentes au seuil 0,01 .
** Effet significatif au seuil 0,01
* Effet significatif au seuil 0,05 . 
riche en acide érucique. Les lots recevant 5 p. Ioo $\left(\mathrm{C}_{5}\right)$ ou 8 p. Ioo $\left(\mathrm{C}_{8}\right)$ de cette matière grasse réduisent très significativement leur poids d'œuf; jaune et blanc sont concernés. Toutefois, en valeur relative, la réduction est plus importante pour le jaune. Lorsque les animaux sont de nouveaux nourris avec le régime témoin (post-expérience) leurs $œ u f s$ tendent à rederenir rapidement identiques à ceux du lot témoin. Par une sorte de compensation, on peut même observer un accroissement de poids si important que les œufs deviennent significativement plus gros que ceux des témoins. C'est le cas du poids de l'cuf du lot $\mathrm{C}_{2}$ qui s'est accru de $3,7 \mathrm{~g}$ alors que pour le lot $\mathrm{C}_{0}$ (témoin) 1'augmentation n'a été que de $2,06 \mathrm{~g}$; de même pour 1'albumen des lots $\mathrm{C}_{2}$ et $\mathrm{C}_{8}$.

Les résultats de l'essai $n^{\circ} 4$ font l'objet du tableau 8 .

\section{TABIEAU 8}

\section{Essai no 4}

(présence ou absence d'acide érucique dans l'huile de colza)

Variation du poids de l'ouf, de l'albumen et du vitellus entre la préexpérience et l'expérience

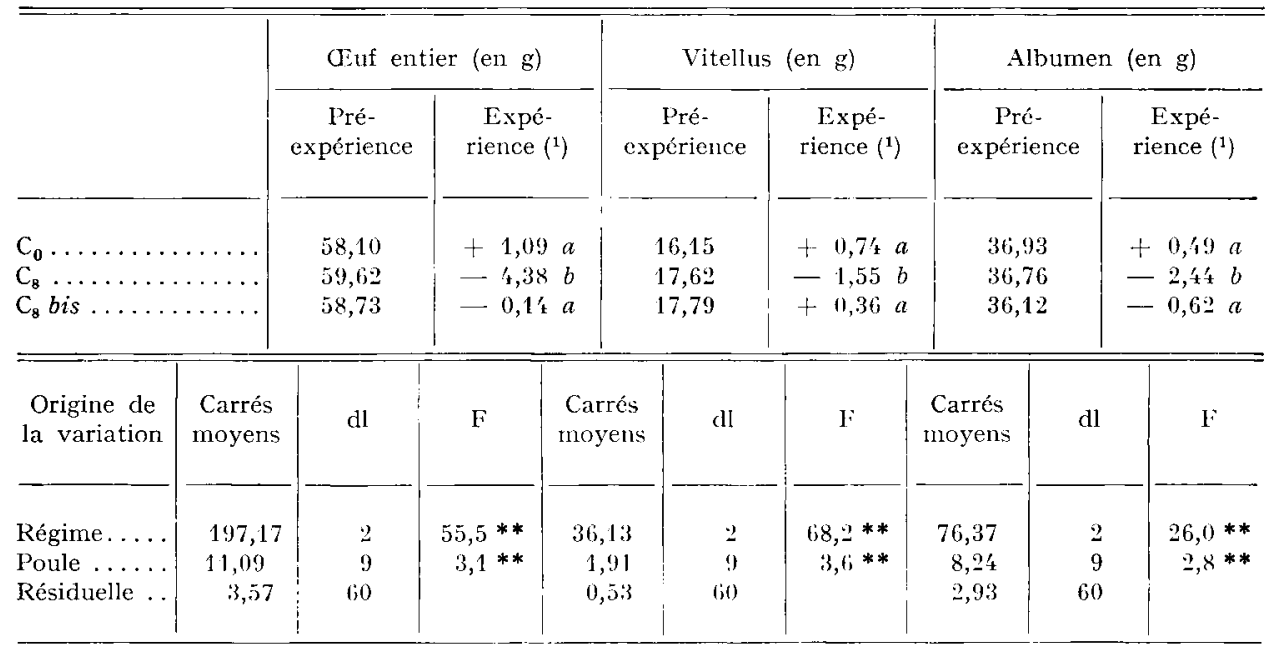

(1) Les valeurs figurant dans cette colonne correspondent aux variations par rapport à la préexpérience; accompagnées de la même lettre, elles ne sont pas significativenent différentes au seuil 0,01 .

** Jiffet significatif au seuil 0,01 * Effet significatif au seuil 0,05 .

L'étude ne comporte cette fois que deux périodes. La comparaison des lots $\mathrm{C}_{0}$ et $\mathrm{C}_{8}$ confirme les observations de l'essai précédent : réduction des poids du jaune et du blanc lorsque les animaux ingèrent 8 p. Ioo d'huile de colza riche en acide érucique. Dans le lot $\mathrm{C}_{8}$ bis consommant 8 p. Ioo d'huile de colza pauvre en acide érucique, cette réduction est très faible et non significative. 


\section{DISCUSSION}

\section{Modification du poids de l'aut due à la nature des acides gras du régime}

Il est bien connu maintenant que la présence de 2 p. Ioo d'acide linoléique est indispensable dans le régime de la Poule pondeuse pour obtenir le poids d'œuf maximum (SHutze et JENSEN, I963; CALVERT I965; LEClERCQ et al., I969). D'autres acides gras ajoutés à la ration peuvent également intervenir en modifiant le poids de l'œuf mais le plus souvent dans le sens d'une diminution; tels sont les acides gras de forme trans et l'acide érucique. Nous avons montré précédemment que les acides gras trans et surtout 1'acide élaïdique réduisent le poids de l'œuf et spécifiquement celui du jaune (LECLERCQ et BLUM, I967). Cet effet aurait pu être en partie imputable à la teneur élevée du régime en matière grasse ou en cellulose; or, la comparaison des lots I et IL avec les lots $A$ et $S$ conduit à rejeter cette hypothèse. La nature des acides gras du régime est donc seule en cause. Nous supposions alors que les acides gras trans induisaient un état de carence secondaire en acide linoléique en augmentant le besoin en cet acide gras indispensable. Cette explication ne peut être retenue dans le cas du poids de l'œuf puisque l'addition d'huile de maïs ne supprime pas l'effet des acides gras trans. On peut penser, par contre, que les acides gras trans qui s'incorporent facilement et massivement à tous les lipides de l'organisme et en particulier à ceux de 1'œuf (LECLERCQ, I967) modifient les propriétés physiques et physico-chimiques des lipoprotéines du vitellus de sorte que leur synthèse ou leur transfert à l'ovaire soient rendus moins aisés. Ceux des autres protéines demeurant inchangés, on comprendrait pourquoi la composition du vitellus se modifie. De plus, l'ovocyte croissant plus lentement, un délai supplémentaire serait nécessaire pour qu'il atteigne une taille suffisante pour être pondu ; cette taille étant toutefois inférieure à ce qu'elle était au cours de la période pré-expérimentale. Cet allongement de la phase de grand accroissement associé à une réduction de taille du vitellus se produit également lors de l'ingestion de thyroxine (LACASSAGNE, I957). Le rythme de ponte ne paraissant en rien modifié par l'ingestion d'huile isomérisée, il faut admettre que le métabolisme de la Poule n'est pas profondément perturbé. D'ailleurs, le retour à une alimentation dépourvue d'acide trans conduit rapidement à un poids d'œuf normal.

Les essais 3 et 4 font ressortir l'effet défavorable sur le poids de l'œuf de l'huile de colza riche en acide érucique. Nous confirmons notre précédente observation (LECLERCQ, Ig68) et celle de KONDRA et al. (Ig68). Il semble bien que ce phénomène soit dû à la présence d'acide érucique. En effet, la réduction est d'autant plus importante que le régime est enrichi en huile de colza. Elle est minime si l'on utilise une huile de colza dépourvue d'acide érucique. On pent, bien sût, imaginer l'existence dans l'huile, d'une substance autre que l'acide érucique et responsable de l'effet toxique ; la présence de cette substance serait liée à celle de l'acide érucique. L'expérimentation avec des acides gras purs devrait permettre de conclure. Le mécanisme par lequel l'huile de colza réduit le poids de l'œuf semble différent de celui de l'huile isomérisée. Certes, dans nos essais, contrairement à ceux d'autres auteurs (KonDRA et al., I968), le taux de ponte n'est pas sensiblement modifié. Cependant, avec l'huile 
de colza, la réduction du poids de l'œuf résulte à la fois de celle du jaune et de celle du blanc. Contrairement à l'huile isomérisée, cette huile ne change pas profondément la composition de l'œuf en acides gras ; 1'acide érucique n'y figure qu'à l'état de traces (LECLERCQ, I968). L'intervention d'un constituant de 1'huile de colza sur la fonction thyroïdienne ne paraît pas fondée (SEL, et HodGson, I963). De même, l'interférence avec l'acide linoléique ou les vitamines semble peu probable (Rocouelin et POTTEAU, r968).

On peut invoquer une mauvaise digestibilité des matières grasses du régime. Or, chez le Poussin (SELL, et Hodgson, I963) comme chez le Dindonneau (Joshi et SELL, I964) la valeur énergétique (énergie métabolisable) de l'huile de colza est tout à fait comparable à celle d'autres huiles (soja). De plus, chez le Rat, le coefficient d'utilisation digestive de l'huile de colza est voisin de celui des autres matières grasses alimentaires (MrddLEToN et CAMPBELL, I958). Enfin, dans nos essais, les consommations d'aliments riches en huile de colza sont voisines de celles des régimes témoins. Cette hypothèse ne peut être retenue que si d'autres facteurs tels que le taux élevé de calcium du régime provoquent une chute importante du C. U. D. de l'acide érucique. Cette chute empêchant 1'absorption d'une quantité suffisante de constituants énergétiques et d'autres nutriments nécessaires à la vitellogenèse comme à l'albuminogenèse, réduirait le poids de l'œuf. Cette réduction serait toutefois suffisamment modérée pour ne pas altérer le rythme de ponte.

Quoi qu'il en soit, nos essais montrent que le poids de l'œuf peut dépendre de la nature des acides gras du régime.

\section{Modification indépendante du poids du jaune et de celui du blanc}

La quantité d'albumen déposée dans l'œuf au niveau de l'oviducte est sensiblement proportionnelle à la taille du vitellus (STURKIE, I965). Cette proportion, caractéristique d'une poule, évolue avec l'âge des animaux et la place de 1'œuf dans la série. Certes, on sait que certains produits (vermifuges) peuvent modifier l'une ou lautre fraction (STURKIE, I965). Nos résultats démontrent que des composants majeurs du régime tels que les graisses ou les acides aminés sont capables de changer le poids du jaune ou du blanc indépendamment l'un de l'autre.

Dans le cas de l'huile isomérisée, on constate que si le poids du jaune est notablement diminué, celui du blanc ne change pas. Autrement dit, la quantité d'albumen sécrétée par l'oviducte ne dépend pas directement de la taille du jaune. On peut expliquer les résultats obtenus en supposant que l'augmentation progressive de la taille du jaune en cours de ponte est le stimulus responsable de l'augmentation parallèle de celle du blanc. En régime normal, le poids du jaune et celui du blanc augmentent tous les deux; chez les poules consommant de 1'huile isomérisée, l'oviducte répond à la réduction de taille du jaune en maintenant sa sécrétion d'albumen sans la diminuer. La relation étroite entre jaune et blanc ne serait donc valable que parce que le poids du jaune s'accroît au cours de la ponte. En présence d'acide érucique, il faut admettre qu'une ou plusieurs modifications importantes affectent le métabolisme de la Poule puisque le poids du blanc se trouve réduit en même temps que celui du jaune.

L'altération de la valeur biologique des protéines concerne tout particulièrement Annales de Biologie animale. - I970. 
l'albuminogenèse. On assiste à une chute du poids du blanc bien que celui du jaune change peu. Le même phénomène peut être constaté lors d'une déficience protéique globale (FISHER, I969). Dans nos essais, le rythme de ponte est alors significativement réduit et la vitellogenèse tout comme 1'albuminogenèse sont touchées ; la réduction est plus importante pour l'albuminogenèse. Il semble donc que dans le cas d'une insuffisance en méthionine et en lysine, il s'instaure une hiérarchie entre les organes : le foie, lieu de synthèse du vitellus, devenant prioritaire par rapport au magnum (priorité vraie ou situation privilégiée pour les synthèses à partir des nutriments provenant du sang porte).

Nos résultats infirment la notion classique selon laquelle, les conditions nutritionnelles ne modifient en rien les proportions de blanc et de jaune dans l'œuf de Poule ('TtrRroine et BELIN, I927).

\section{Modification de la composition du jaune et du blanc}

A la suite de TERroine et BEIIN (I927), on a admis et l'on admet encore que les teneurs du blanc et du jaune en lipides et protéines sont fixes et indépendants de l'alimentation ('TERROINE et BELIN, I927). Cette notion n'est pas confirmée dans nos essais. En effet, les acides gras trans du régime modifient la composition du jaune. Sa teneur en protéines augmente et celle des lipides diminue. Nous avons plus haut tenté d'expliquer ces résultats par une modification de la composition des lipoprotéines. Il est intéressant de constater que la carence en acide linoléique, qui réduit aussi le pourcentage de jaune dans l'œuf, provoque parallèlement une diminution de la teneur du vitellus en lipides (CALVERT, I967), et que par ailleurs, certains auteurs attribuent l'effet favorable de l'acide linoléique sur le poids de l'ouf à l'intervention de cet acide gras dans la synthèse et la structure des lipoprotéines (JENSEN, Ig68).

Nous avons constaté de plus que la déficience du régime en méthionine et en lysine conduit à des changements profonds de la composition du jaune et du blanc : la teneur en eau de ces deux fractions est plus élevée. Dans le vitellus, la diminution de la teneur en lipides sans modification de celle des protéines peut s'expliquer par une réduction plus importante de la synthèse des lipoprotéines que des protéines solubles. Dans l'albumen la réduction de la teneur en protéines, s'ajoutant à celle du poids du blanc, témoigne d'une altération profonde de la protéinogenèse au niveau du magnum. L'accroissement du taux d'hydratation du blanc s'expliquerait alors soit par une modification du transit minéral soit par un changement dans les proportions des diverses protéines qui conditionnent l'hydratation de l'albumen. Une observation semblable a été faite par MARCH et BIELY (Ig63) avec des régimes déséquilibrés par excès de lysine.

En conclusion, il existe une certaine indépendance entre les différents processus de formation des constituants de l'œuf : indépendance entre les lieux de synthèse qui peut s'expliquer par une hiérarchie entre les organes (foie, magnum), indépendance au sein d'un même organe entre les synthèses des divers constituants (glycoprotéines, lipoprotéines, holoprotéines). Des facteurs nutritionnels qui réduisent le poids de l'œuf entier peuvent ne pas affecter de la même façon le jaune et le blanc tant dans leurs proportions relatives que dans leur composition. 


\section{SUMMARY}

\section{NUTRITIONAL FACTORS WHICH MODIFY THE WEIGHT OF EGGS AND THEIR CONSTITUENTS} The effect of different nutritional factor on the weight of eggs and their composition was
studied.

Trans fatty acids in the diet caused a large and rapid reduction in the weight of the egg yolk (Groups I and IL in tables 4 and 5). Duration of the phase of growth of the ovocyte was mean whileextended (table 5). These effects were independent of the linoleic acid content of the diet (compa rison between groups I and IL). They were accompanied by a change in the composition of the yolk : increase in protein content with concentration of lipids remaining constant (table 6). The trans fatty acids would interfere with the synthesis or the transfer of lipoproteins of the yolk, profoundly altering their composition.

A moderate deficiency of methionine and lysine (group VB) reduced the weight of the egg, mainly in the albumen; weight of the yolk did not diminish. Moreover, the water content of yolk and white were markedly increased (table 6). Thus deficiency of essential amino acids affects formation of white more than formation of yolk.

Inclusion of rapeseed oil rich in erucic acid in the diet of laying hens leads within a few days to a reduction in egg weight involving both white and yolk. This effect is seen with a small amount in the diet (table 7). It disappears in a few days when the rapeseed oil is withdrawn. It does not occur if rapeseed without erucic acid is used (table 8, comparison between groups and $\mathrm{C}_{8}$ and $\mathrm{C}_{8}$ bis) This phenomenon can only be explained by a reduction in digestibility of the rapeseed oil, causing a loss of the nutrients necessary for egg formation. The high calcium content of the diet could have been responsible for the poor digestibility.

There is a certain independence among the different processes of formation of the constituents of the egg : independence between the sites of synthesis (magnum and liver) and independence within the same organ between synthesis of different constituents (lipoproteins, glycoproteins...). Nutritional factors which alter egg weight cannot operate in the same way on the yolk and the white.

\section{RÉFÉRENCES BIBLIOGRAPHIQUES}

Calvert C. C., I965. Lipid metabolism in laying hen : essential fatty acids. Proc. Cornell Nutr. Conf. Feed Manif., I04-II2.

Calvert C. C., I967. Studies on hatchibility of fertile eggs from hens receiving a linoleic acid deficient diet. Poult. Sci., 46, 967-973.

JENSEN L. S., I 968 . Vitamine E and essential fatty acids in avian reproduction. Fed. Proc., 27, 9I4-9I9

Joshi S. K., Sell J. L., r964. Comparative dietary value of soybean oil, sunflower oil, rapeseed oil and animal tallow forturkey poults. Canad. J. anim. Sci., 44, 34-38.

LACAssagne L., I957. Action de la dl-thyroxine sur la durée d'évolution de la phase de grand accroissement du follicule chez la Poule domestique. C. R. Acad, Sci., 245, I830-1832.

Lacassagne L., 1957. Contribution à l'étude de la phase de grand accroissement des follicules chez la Poule domestique. Ann. Zootech., 6, 85-93.

LECLERcQ B., r967.Utilisation de l'acide élaïdique pour l'étude du métabolisme des lipides chez la Poule pondeuse. Arch. Sci. Physiol., 21, 299-322.

Leclerce B., Blum J. C., I967. Utilisation par la Poule pondeuse de l'huile d'arachide isomérisćeSes effets sur la genèse et la composition des réserves vitellines. Ann. Biol. anim. Bioch. Biophys., $\boldsymbol{\gamma}$, 303-3 5 .

LeclercQ B., I968. Utilisation de l'huile de colza par la Poule pondeuse; son influence sur la genèse et la composition des lipides de l'œuf. C. R. Acad. Sci., 267, 2235-2237.

Leclerco B., Blum J. C., Delpech P., I969. Influence du régime maternel sur la croissance du jeune poussin. Effets d'une déficience en acide linoléique. Ann. Biol. anim. Bioch. Biophys., 9, r91-204.

MARCH B. E., Biely J., 1963. Control of egg size in chickens through dietary amino acid balance. Nature, 200, 702-703. 
Middleton E. J., Campbell J. A., I958. The détermination of the available caloric content of rapeseed oil by rat growth. Cand. J. Biochem. Physiol., 36, 203-208.

Rocquelin G., Potteau B., ig68. La valeur nutritionnelle et les effets physiopathologiques de l'huile de colza. Influence de sa teneur en acide érucique. Ann. Nutr. Alim., 22, I9I-244.

Sell J. L., Hodgson G. C., I962. Comparative value of dietary rapeseed oil, sunfower oil, soybean oil and animal tallow for chickens. J. Nutr., r6, I I3-1 I8.

Sturkie P. D., I965. In Avian Physiology. Comstock Publishing Associates. Cornell Univ. Press Ithaea N. Y.

Shutze J. V., Jensen L. S., I963. Influence of linoleic acid on egg weight. Poult. Sci., 42, 92I-924.

TERroIne E. F., BeLIN P., r927. Influence de l'alimentation sur la composition quantitative de l'œuf de poule. Bull. Soc. Chim. Biol., 2, 1074-1084. 